\title{
3 Research Soure

\section{WNT pathway alteration is related to dysplastic cortical tissue and not to adjacent tissue of patients with FCD and refractory epilepsy}

Daniel Marinowic ( $\nabla$ daniel.marinowic@pucrs.br)

PUCRS: Pontificia Universidade Catolica do Rio Grande do Sul

Gabriele G. Zanirati

PUCRS: Pontificia Universidade Catolica do Rio Grande do Sul

Fernanda Majolo

UNIVATES: Universidade do Vale do Taquari

Fernando A. C. Xavier

PUCRS: Pontificia Universidade Catolica do Rio Grande do Sul

Felipe V. F. Rodrigues

PUCRS: Pontificia Universidade Catolica do Rio Grande do Sul

Eliseu P. Neto

PUCRS: Pontificia Universidade Catolica do Rio Grande do Sul

André L. F. Palmini

PUCRS: Pontificia Universidade Catolica do Rio Grande do Sul

José G. de Abreu

UFRJ: Universidade Federal do Rio de Janeiro

Denise C. Machado

PUCRS: Pontificia Universidade Catolica do Rio Grande do Sul

Jaderson C. da Costa

PUCRS: Pontificia Universidade Catolica do Rio Grande do Sul

\section{Research Article}

Keywords: focal cortical dysplasia, beta-catenin, wnt pathway, epilepsy

Posted Date: December 3rd, 2021

DOI: https://doi.org/10.21203/rs.3.rs-1128423/v1

License: (c) (i) This work is licensed under a Creative Commons Attribution 4.0 International License.

Read Full License 


\section{Abstract \\ Background}

Focal cortical dysplasia (FCD) is a malformation of the cortical development that cause medical refractory seizures and the only treatment may be surgical resection of the affected area of the brain. People affected by FCD may present seizures of variable severity since childhood. The physiopathology of the disease is not yet understood, however it is known that several genes alterations may play their role. The WNT/ $\beta$-catenin pathway is associated with cell transformation and migration and for this reason may be crucial for understanding FCD's aetiology. The aim of this study was to explore genes related to the WNT/ $\beta$-catenin pathway in patients with FCD type II.

\section{Methods}

Dysplastic tissue and tissue adjacent to the primary dysplastic lesion of patients with FCD type II were obtained from two patients who underwent surgical treatment. The analysis of the relative expression of genes was performed by a qRT-PCR array containing 84 genes related to the WNT pathway.

\section{Results}

In patient 1 , the analysis showed a difference in the expression of seven genes, demonstrating an increase in AXIN2, FRAT2, FZD9, KREMENI and PP2R1A genes and a reduction in CSNK1G3 and PPP2CA genes in dysplastic tissue. In patient 2, the analysis showed increased expression of CSNK1A1, FZD4 and PPP2CA genes, as well as reduced of CTNNBIP1 gene in dysplastic tissue.

\section{Conclusion}

Dysregulation in the expression of genes that control the receptors of the WNT pathway keeps it in an inactivated state. Therefore, a eventual manipulation of this pathway focusing on its activation may influence molecular manifestations underlying the epileptogenic status in injured brain tissue, which could act as a therapeutic alternative to FCD type II. The WNT/ $\beta$-catenin signaling pathway is crucial for the control of embryonic development, which takes place through the regulation of cell differentiation, migration and proliferation, and apoptosis process.

\section{Introduction}

Focal Cortical Dysplasia (FCD) is a malformation of cortical development (MCD) characterized by abnormalities in the cerebral cortex, which results from alterations in cellular migration and differentiation processes during neurodevelopment (1). It is the most common cause of refractory epilepsy in children, and one of the most common causes of it in adults (2). In 2004, Palmini et al. (3) 
classified FCD according to the presence or absence of cellular abnormalities, such as disphormic neurons or balloon cells, as well as by the presence of cortical dyslamination. A new classification system was proposed by Blumcke et al. in 2011 (4), which divided FCD into three categories, now including abnormalities such as tumor and/or ischemic injury associated with the main lesion in a third FCD type.

Although still highly prevalent, FCD's exact etiology remains unknown. Many genes have been reported to be associated with the disease, as changes in their expression or mutations in their sequences may cause alterations in cell-signaling pathways which are key factors during development, like the AKT, mTOR and $\mathrm{PI} 3 \mathrm{~K}$ pathway(5). The WNT/ $\beta$-catenin is also a signaling pathway crucial for controlling embryonic development through regulation of cellular differentiation, migration, proliferation and apoptosis. Since $\beta$ catenin is a protein responsible for cell-cell adhesion and a component of the cadherin protein complex $(6,7)$, the WNT pathway may also be involved in the etiology of FCD.

The WNT/ $\beta$-catenin plays an essential role during embryonic development and tissue homeostasis by regulating cell proliferation and differentiation1. Abnormalities of Wnt/ $\beta$-catenin signaling is are associated with organogenesis defects, cancer, and degenerative diseases 2 . In the central nervous system (CNS), the Wnt pathway plays a role in synaptic transmission 3 , and its activation enhances longterm potentiation (LTP), while its inhibition impairs LTP 4. Recently the WNT/ $\beta$-catenin pathway has gained special attention as it is associated with epilepsies. Its participation in the adult neurogenesis induced by seizures, as well as the hippocampal sclerosis characteristic of temporal lobe epilepsies, presents the WNT pathway as a potential therapeutic target for future antiepileptogenic treatments(8). Here, dysplastic tissue and adjacent to the main dysplastic lesion were obtained from two Focal Cortical Dysplasia type Ilb patient with the aim of studying genes with similar expressions between tissues by qRT-PCR analysis from an array containing 92 primers related to WNT pathway genes.

\section{Methods}

\section{Ethics statement}

This study was approved by the Committee of Research Ethics of the Pontifical Catholic University of Rio Grande do Sul (CAAE: 19776619.9.0000.5336 approval number: 3.577.035). Written consentment was obtained from the parents of Patients 1 and 2 (which are underage) enrolled in the study, according to Brazil Resolution $n^{\circ} 466 / 12$.

\section{Patients and sample collection}

The sample was collected during the surgical procedure. The lesion dysplastic tissue were removed according to the surgical plan for each patient. The non-lesion tissues (adjacent to the lesion) were collected inevitably due to the need to reach the area to remove the damaged tissue. All samples were immediately placed in RNA Later solution (Thermo Fisher Scientific, Massachusetts, EUA) and storage at $-80^{\circ} \mathrm{C}$ until the protocol of RNA extraction. 
The characteristics of each patient are described in Table 1.

Table 1

Clinical and laboratory characteristics of each patient enrolled in the study.

\begin{tabular}{|c|c|c|}
\hline & Patient 1 & Patient 2 \\
\hline Age & 17 years & 13 years \\
\hline Genre & Female & Female \\
\hline $\begin{array}{l}\text { Refractory } \\
(\mathrm{Y} / \mathrm{N})\end{array}$ & $\mathrm{Y}$ & $\mathrm{Y}$ \\
\hline First Seizure & At 10 years of age & At 5 years of age \\
\hline Comorbidity & - & Dyslexia \\
\hline \multirow[t]{2}{*}{ EEG region } & & $\begin{array}{l}\text { Suggestive of focal epilepsy with discrete } \\
\text { diffuse disorganization of the base } \\
\text { activity. }\end{array}$ \\
\hline & & $\begin{array}{l}24 \mathrm{hrs} \text { VEEG is compatible with focal } \\
\text { epilepsy involving the posterior quadrants } \\
\text { of both hemispheres, with electrographic } \\
\text { onset crises in the occipital region on the } \\
\text { right, which most likely represents a false } \\
\text { electronographic lateralization. }\end{array}$ \\
\hline $\begin{array}{l}\text { Family } \\
\text { history }\end{array}$ & $\begin{array}{l}\text { Maternal uncle with epilepsy hc of } \\
\text { difficult control. }\end{array}$ & \\
\hline $\begin{array}{l}\text { Clinical } \\
\text { features }\end{array}$ & $\begin{array}{l}\text { Cephalic version to the right, } \\
\text { accompanied by ocular version. Groan } \\
\text { and head fall (vertical bending) }\end{array}$ & \\
\hline $\begin{array}{l}\text { Current } \\
\text { Medications }\end{array}$ & $\begin{array}{l}\text { Trileptal, Urbanil, Lacosamidal (Vimpat) } \\
\text { and CBD. }\end{array}$ & Clobazam and Trileptal. \\
\hline $\begin{array}{l}\text { Post- } \\
\text { Surgery } \\
\text { Medications }\end{array}$ & $\begin{array}{l}\text { Clobazam, Oxcarbazepine, and } \\
\text { Lacosamide. }\end{array}$ & Clobazam and Oxcarbazepine. \\
\hline $\begin{array}{l}\text { Treatment } \\
\text { performed }\end{array}$ & $\begin{array}{l}\text { Resection of right upper frontal rotation } \\
\text { without intercurrences. }\end{array}$ & \\
\hline $\begin{array}{l}\text { Magnetic } \\
\text { Resonance }\end{array}$ & $\begin{array}{l}\text { Blurring and alteration of the cortical- } \\
\text { subcortical interface of the posterior } \\
\text { segment of the upper right frontal } \\
\text { gyrus, compatible with malformation of } \\
\text { the cortical development. Simple cyst } \\
\text { of pineal, measuring about } 1.3 \mathrm{~cm} \text {. }\end{array}$ & $\begin{array}{l}\text { Signal change and lack of definition of the } \\
\text { cortico-subcortical interface of the left } \\
\text { occipital lobe, a finding probably related to } \\
\text { malformation of cortical development. }\end{array}$ \\
\hline
\end{tabular}

\section{RNA extraction and generation of cDNA library}


The total RNA was extracted from the surgically resected brain tissues using the SV-Total RNA Isolation System (Promega, Madison, Wisconsin, EUA) following manufacturer protocol. The RNA extracted was converted in cDNA using VILO MasterMix (Thermo Fisher Scientific, Massachusetts, EUA). cDNA was quantified in a NanoDrop to calculate the concentration needed for the qRT-PCR analysis.

\section{Relative gene expression by Super Array technique}

The cDNA generated from the samples were added to a TaqMan ${ }^{\circledR}$ Array 96-Well Plate for the Human WNT Pathway. This super array contains 92 complementary primers of regions of genes relationship to yhe WNT pathway and 4 endogenous genes control. The samples were amplified from the initial amount of $20 \mathrm{ng}$ of cDNA for each sample. Real-time PCR was performed using StepOne Plus (Thermo Fisher Scientific, Massachusetts, EUA) equipment using the PowerUp Master Mix kit (Thermo Fisher Scientific). Results were analyzed in Thermo Fischer Connect Platform for the generation of heatmaps.

\section{Relative gene expression analysis}

Relative gene expression analysis was performed using the $\Delta \Delta C T$ methodology, comparing dysplastic and adjacent cortical tissues individually (Patient 1 and Patient 2) and in a grouped way (Group) using the GAPDH gene like housekeeping. A cut off was assigned using as a basis for individual analysis the genes that showed fold change greater or less than 5 times in the comparison between healthy and diseased tissues. The analysis of the results was performed using the Thermo Fischer Connect software (Thermo Fischer Scientific).

\section{Results}

\section{Gene expression analyses}

The limitation of this study is that some genes had undetermined results in the super array analysis mainly in Patient 1. A heatmap illustrating the expression patterns of WNT pathway genes from dysplastic and adjacent cortex brain. Each horizontal row represents the same gene product and each vertical row the same tissue. The fluorescence ranges from high (green - maximum) to low (red minimum) is indicated by the colored bar and reflects the degree of fluorescence intensity/gene expression. For individual analysis of genes, we used a cut off for genes that showed fold change greater or less than 5 times in comparison between healthy and diseased tissues. The CTs values of genes inclunced in this analisys criteria are describe in Table 2.

Tabela 2. CT values used to calculate gene expression (fold change) of the genes that showed alterations greater than 5 times their relative expression. $H G=$ Housekeeping Gene 


\begin{tabular}{|c|c|c|c|c|c|c|c|c|}
\hline & $H G$ & AXIN2 & FRAT2 & FZD9 & KREMEN1 & PPP2R1A & CSNK1G3 & PPP2CA \\
\hline \multicolumn{9}{|l|}{ Patient 1} \\
\hline Heathy tissue & 24.97 & 30.94 & 31.97 & 33.97 & 33.95 & 27.94 & 29.95 & 26.94 \\
\hline \multirow[t]{2}{*}{ Dysplasic tissue } & 37.97 & 40.95 & 40.92 & 41.91 & 41.97 & 39.96 & 42.03 & 41.99 \\
\hline & $H G$ & CSNK1A1 & FZD4 & PPP2CA & CTNNBIP1 & & & \\
\hline \multicolumn{9}{|l|}{ Patient 2} \\
\hline Heathy tissue & 23.96 & 28.95 & 30.90 & 27.95 & 28.96 & & & \\
\hline \multirow[t]{2}{*}{ Dysplasic tissue } & 25.96 & 30.95 & 33.95 & 28.94 & 38.99 & & & \\
\hline & $H G$ & AXIN2 & CTNNBIP1 & KREMEN1 & KREMEN2 & SFRP2 & WNT8B & \\
\hline \multicolumn{9}{|l|}{ Group } \\
\hline Heathy tissue & 24.46 & 29.94 & 29.45 & 32.45 & 42.02 & 29.89 & 39.92 & \\
\hline Dysplasic tissue & 31.96 & 36.96 & 40.47 & 38.47 & 42.01 & 31.00 & 38.93 & \\
\hline
\end{tabular}

\section{Patient 1 analysis}

The comparison of $\Delta \mathrm{CT}$ 's between healthy and dysplastic tissues from patient 1 showed a very different dispersion, evidencing a profile of greater difference between behavior of the analyzed genes (Figure 1).

When analyzing the values from the $2^{-\triangle \Delta C T}$ calculation, the comparison between lesion and adjacent tissues in patient 1 showed differences in the expression of 7 genes when applying the cut off criterion established in this study. AXIN2, FRAT2, FZD9, KREMEN1 and PPP2R1A genes showed an increase in relative expression and CSNK1G3 and PPP2CA genes showed a reduction in relative expression values, controlling healthy tissue and dysplastic brain tissue as the target of investigation. In dysplastic brain tissue, there was a 53.74-fold increase in the expression of the Axin-related protein gene (AXIN2) when compared to healthy tissue. The FRAT2 gene (FRAT Regulator of WNT Signaling Pathway 2), FZD9 (Frizzled Class Receptor 9), KREMEN1 (kringle containing transmembrane protein 1) and PPP2R1A (Protein Phosphatase 2 Scaffold Subunit Alpha) showed an increase of $6.93,7.55,13.45$ and 33.13 times, respectively, in brain tissue with dysplasia. There was a reduction of 5.02 times in CSNK1G3 (Casein Kinase 1 Gamma 3) gene expression and also a reduction of 33.12 times in PPP2CA (Protein Phosphatase 2 Catalytic Subunit Alpha) gene expression in diseased brain tissue compared to healthy tissue.

\section{Patient 2 analysis}

Patient 2 showed a more homogeneous distribution of delta CT values when comparing the values of healthy brain tissue with the affected one (Figure 1), thus characterizing a greater similarity in the genes behavior. When $2^{-\triangle \Delta C T}$ values were generated and the cut off established, patient 2 presented a difference in the expression of 4 genes. There was a 319.57-fold increase in expression of the CSNK1A1 gene (Casein Kinase 1 Alpha 1) in dysplastic brain tissue compared to the non-dysplastic. The FZD4 gene (Frizzled Class Receptor 4) showed a 12.7-fold increase and the PPP2CA gene (Protein Phosphatase 2 Catalytic Subunit Alpha) a 6.99-fold increase in dysplastic tissue. There was a very important reduction in 
the expression of the CTNNBIP1 gene (catenin beta interacting protein 1), and this gene was 316.26 times less expressed in dysplastic brain tissue compared to healthy brain tissue in patient 2 .

\section{Group analysis}

By analyzing the gene expression results of the two patients together and comparing brain tissue with cortical dysplasia and healthy tissue, it was possible to identify changes in the expression of AXIN2, CTNNBIP1, KREMEN1, KREMEN2, SFRP2 and WNT8B genes. There was a 7.13-fold increase $(p=0,254)$ in AXIN2 gene expression in brain tissue with dysplasia compared to healthy tissue. There was also an increase in the expression of KREMEN1 and KREMEN2 genes of $5.03(p=0,174)$ and $6.38(p=0,0008)$ times respectively. Furthermore, the WNT8B gene showed a 10.92-fold increase $(p=0,282)$ in relative expression. Two genes showed reduced relative expression in diseased brain tissue. The CTNNBIP1 gene showed a 33.89-fold reduction $(p=0,179)$ in relative expression and the SFRP2 (Secreted Frizzled Related Protein 2) gene showed a very expressive reduction of 206.81 -fold $(p=0,368)$.

\section{Discussion}

Different genes have already been associated with cortical malformation, including FCD type II, directly related to cell proliferation, cell cycle regulation, neuronal migration, late cortical organization and structure and function of the cytoskeleton (9-12). Alterations in these genes can have consequences for CNS, including the place where the cortex can be affected (5).

In this work, we explored an WNT pathway genes array from samples obtained from dysplastic tissue and from the tissue adjacent to the main dysplastic lesion.

Both patients were consistent to FCD type II, with medically refractory seizures. Histopathology presents cortical dyslamination and large, dysplastic neurons, with balloon cells(5).

Both patients are female and with similar ages, so, there may be similarities in responses of cells. We find it more correct to explore and work on the results of Patient 1, Patient 2, and both patients as a group, separately.

\section{Patient 1}

The increased relative expression of KREMEN1 gene by 13.45 times may influence the blocking of activation of the WNT pathway. The KREMEN1 gene encodes the dickkopf homolog 1 high-affinity transmembrane receptor that functionally cooperates with DKK1 and 2 to block signaling in the WNT/beta-catenin pathway. The DKK receptor coupled to LPR5/6 acts as a major inhibitor of WNT pathway activation, not allowing LPR5/6 to bind to Frizzled pathway receptors. The KREMEN1 protein is still associated with the mechanism of endocytosis of LRP5/6 receptors, which in dysplastic brain tissue could further contribute to blocking WNT activation. Physiologically, the reduction or absence of DKK1 potentiates WNT signaling by keeping LRP5 or LRP6 in the cell membrane. The consequence of this 
process may be an increase in intracellular $\beta$-catenin and the need for degradation, given the nonactivation of WNT pathway and phosphorylation of $\beta$-catenin (13).

The dysplastic tissue of patient 1 also showed a 53.74-fold increase in the expression of the AXIN2 gene, which implies that there is an increase in this transcript in the intracellular environment of this tissue. The AXIN2 protein plays an important role in regulating the stability of $\beta$-catenin in the WNT signaling pathway. The AXIN2 protein is responsible together with CK1, GSK and APC to phosphorylate $\beta$-catenin. Phosphorylated $\beta$-catenin undergoes the process of $\beta \operatorname{TrCP}$-mediated ubiquitination, increasing proteostasis and degrading $\beta$-catenin in the cytosol (14). This AXIN2-mediated mechanism is characteristic of the non-activated WNT pathway where the main objective is the degradation of $\beta$-catenin not used as a transcription factor. The $\beta$-catenin dysregulation is an important event in the genesis of a number of malignant diseases. Control of AXIN2 mediated $\beta$-catenin phosphorylation is essentially important for normal remyelination kinetics, thus AXIN2 is being observed as a regulatory and therapeutic target in brain lesions and newborn remyelination (15).

The dysplastic brain tissue of patient 1 still showed a 7.55-fold increase in the expression of the FZD9 gene. This gene is member of the frizzled gene family that encode transmembrane domain proteins that are receptors for WNT signaling proteins. FZD9 is coupled to the $\beta$-catenin canonical signaling pathway (WNT2) and its activation promotes the inhibition of the GSK3 kinase, $\beta$-catenin nuclear accumulation and activation of WNT target genes (16). The low activation of receptors of the WNT pathway in this tissue, due to alterations in the $\beta$-catenin degradation pathway and DKK blockade, can lead the cell to establish a compensatory mechanism seeking to increase the number of FZD receptors in the cell membrane. Activation of FZD9 has a negative regulatory role in the clustering of acetylcholine receptors (AChR) (17).

Furthermore, the 33.13-fold increase in the expression of the PPP2R1A gene, responsible for the negative control of cell growth and division, suggests that dysplastic tissue presents a response compatible with a control in cell division rates by transcribing a gene that blocks cycle and division, thus appearing to be an unregulated compensation referring to possible hyperactivity of this tissue. In another way, the PPP2CA gene showed a 33.12-fold reduction in relative expression, being also a gene responsible for the negative control of the cell cycle(18).

In short, the molecular presentation of patient 1 allows us to suggest possible alterations in the dysplastic tissue compared to the tissue adjacent to the lesion..

\section{Patient 2}

Dysplastic brain tissue from patient 2 showed a smaller amount of genes with alterations in their relative expression compared to patient 1. On the other hand, some mechanisms have similar pathways related to non-activation of the WNT pathway. The 319.57-fold increase in CSNK1A1 also represents a possible increase in $\beta$-catenin degradation because this gene transcribes the CK1 protein, which is also associated with GSK, AXIN2 and APC, and promotes $\beta$-catenin phosphorylation and ubiquitination and its 
consequent degradation (19). As in patient 1, there was a 12.7-fold increase in FZD9 expression in dysplastic tissue. As already mentioned, FZD9 is coupled to the $\beta$-catenin canonical signaling pathway (WNT2) and its activation promotes the inhibition of the GSK3 kinase, $\beta$-catenin nuclear accumulation and activation of WNT target genes. In patient 2, there was a 316.26-fold reduction in the expression of CTNNBIP1, a gene involved in the inhibition of transcription factors in cells after activation of the WNT pathway. The protein translated by this gene is a negative regulator of the WNT signaling pathway. CTNNBIP1 prevents the interaction of TCF/LEF proteins that coupled to intranuclear $\beta$-catenin after activation of the WNT pathway, promote the transcription of genes responsible for maintaining growth, proliferation, migration, and cell cycle control(20). The reduction in the expression of this gene may lead to lack of control regarding the coupling of non-phosphorylated $\beta$-catenin after activation of the WNT pathway, as well as it may be a cellular response to a possible accumulation of phosphorylated $\beta$-catenin that tries to be controlled by the increase in CSNK1A1 and non-phosphorylated that tries to be used as a transcription factor together with TCF/LEF due to the increased interaction of these factors mediated by the reduction in the expression of CTNNBIP1.

\section{Group analysis}

Analyzing the results of the relative expression when grouping patient 1 with patient 2 and comparing the injured tissue with the adjacent tissue, the results follow suggestive of an increase in cytoplasmic $\beta$ catenin degradation by phosphorylation and inactivation of the WNT pathway mediated by an increase in DKK on LPR5/ 6 receptor. There was a 7.13-fold increase in the expression of AXIN2, involved in the phosphorylation and ubiquitination of $\beta$-catenin and its consequent degradation in the non-active form of the WNT pathway. There was also an increase in KREMEN1 and KREMEN2 of 5.03 and 6.38 times, respectively, which induces the blocking of WNT activation by the increase of DKK, causing the nonactivation of LPR5/ 6 receptors and bound to the Frizzled WNT receptors. As in patient 2, analyzing in a grouped way, there was a 33.89-fold reduction in the expression of the inhibitor of the binding between $\beta$ catenin and the transcription factor TCF/LEF, also appearing to be a compensation mechanism for transcription of genes related to activation of the WNT pathway which in diseased tissue appears not physiologically activated.

\section{Hypothesis about the WNT pathway in brain tissue with focal cortical dysplasia}

Overall, this study revealed some changes that may be suggestive of the refractory epileptogenic phenotype associated with diseased tissue in patients with Focal Cortical Dysplasia type II. Based on the results obtained in this study and in the current literature, the hypothesis of the involvement of the WNT pathway in the mechanisms associated with high excitability of the diseased tissue can be constructed and alternative suggestions for mediators for the control of seizures can be considered based on blockade, silencing or stimulation of transcription of genes involved in the WNT pathway.

The WNT pathway has recently been associated with epilepsies as it participates in the pathway in neurogenesis induced by seizures, as well as in the essential characteristic of the pathology, being 
observed as a potential therapeutic target for future antiepileptogenic treatments (15).

Specifically, in relation to epilepsy, the focus of WNT signaling involvement has been in relation to seizure-induced neurogenesis and neuronal death in the acute and chronic phases of the seizure. In different experimental models of epilepsy, increases in the expression of WNT signaling components were evidenced and they were associated with an increase in neurogenesis and neuronal death, commonly observed after seizures (21-23) .

Some protective and beneficial mechanisms related to protection against neuronal death are observed after modulation of the WNT pathway in models of cerebral ischemia and induced seizures, with the WNT pathway being a non-aberrant repair control mechanism of neurogenic brain areas (24).

The increase in DKK signaling induced by the increase in the KREMEN 1 and 2 gene transcript can induce inhibition of the WNT pathway by blocking LRP5/5 receptors and their consequent non-binding to Frizzled receptors, thus keeping cells in the dysplastic tissue, in a not activated state of the pathway. It was demonstrated in an experimental model that when using an antagonist of the endogenous DKK1 inhibitor, there was a protection against the development of hippocampal sclerosis, a finding that is closely related to the problems of temporal lobe epilepsy (25). Therefore, by antagonizing the DKK1 inhibitor, there is protection against the formation of hippocampal sclerosis after a seizure. This process using the DKK1 antagonist, which is an inhibitor of the WNT pathway, probably increases the binding of LPR5/6 to Frizzled receptors, leaving the WNT pathway in a more active state in these cells and this activation mediates protection to this tissue. When comparing dysplastic brain tissue with the adjacent one, we suggest that there is an increase in DKK blocking the WNT pathway in this diseased tissue, not allowing an adequate homeostasis necessary to promote a better post-seizure condition. WNT blockade may be related to the worsening of the epileptogenesis process since reduced neurogenesis in the chronic phase of epilepsy can potentially exacerbate tissue hyper excitability over time due to a reduction in the number of inhibitory neurons and their generated connections, consequently keeping the tissue more excitable $(26,27)$.

Another important finding in the molecular profile of brain tissue with FCD type II was the increased expression of AXIN2 and CSNK1A1 genes, both involved in the mechanism of phosphorylation and ubiquitination of $\beta$-catenin and its consequent cytoplasmic degradation. One of the first studies to demonstrate the role of $\beta$-catenin in tissue after seizures elucidated that seizures induced by electroconvulsive technique increase the expression of $\beta$-catenin and ligand WNT2 in new neurons generated in the hippocampus of rats (21). Therefore, the seizure seems to promote an increase in cytoplasmic $\beta$-catenin, to reorganize the neurogenic environment and promote the generation of new neurons mediated by the activation of the WNT pathway and its consequent transcription of genes related to homeostasis and cellular control. It was demonstrated in a study using a murine model of WNT signaling reporter mouse line (Topgal mice) that an increase in $\beta$-catenin levels occurs in reactive astrocytes after induced cortical ischemia, with increased $\beta$-catenin expression being associated with an increase in susceptibility to seizures in these models of ischemia (28). Here, we observed an increase in 
AXIN2 and CSNK1A1 genes which may be related to an attempt mechanism of $\beta$-catenin degradation increased by the convulsive aspect of dysplastic cortical tissue. Furthermore, the increase in $\beta$-catenin degradation may be an attempt at degradation not only due to the increase commonly observed in epileptogenic tissues but also due to the non-activation of the WNT pathway and its consequent need to eliminate cytoplasmic $\beta$-catenin since it is not used in unphosphorylated form as a transcription factor coupled to CPB and TCF/LEF.

Furthermore, we observed an increase in the FZD9 transcript, suggesting that cells from tissue with FCD are responding to a feedback mechanism in an attempt to generate a greater number of Frizzled receptors to compensate for the inactivity of the WNT pathway in this tissue. The generation of new receptors may be a response of diseased tissue cells to the accumulation of cytoplasmic $\beta$-catenin and the consequent increase in its degradation, in addition to blocking the activation of WNT mediated by the increase in available DKK.

The particular condition of patient 2 was a very significant reduction in the expression of the CTNNBIP1 gene. This gene is related to the interaction between $\beta$-catenin and the intranuclear TCF/LEF protein that will serve as a transcription factor in the activation of genes related to the WNT pathway. CTNNBIP1 is an inhibitor of this interaction and in patient 2 we observed an increase of more than 300 times the expression of this gene. The high concentration of cytoplasmic $\beta$-catenin can promote the reduction of the CTNNBIP1 inhibitor so that the interaction of nuclear transcription factors occurs. This fact leads to a suggestion that the WNT pathway could be more active in this tissue due to the expressive reduction of the gene that inhibits the interaction of non-phosphorylated $\beta$-catenin, which occurs only after the activation of the WNT pathway mediated by FZD receptors. We can also suggest that the expected increase in cytoplasmic $\beta$-catenin may alter the degradation mechanisms of the non-active pathway as well as seeking a balance in the active pathway mediated by the reduction in the expression of the negative controller gene of the nuclear interaction of non-phosphorylated $\beta$-catenin and its consequent use in the activated WNT pathway.

\section{Conclusion}

By comparing the expression of genes related to the WNT pathway using dysplastic brain tissue compared to healthy tissue from the same patient, we concluded that possible molecular mechanisms might be associated with poor prognosis of seizure control as well as patients' return to refractoriness after surgical resection of the affected areas. We found a dysregulation in the expression of genes that control the blocking of LPR5/ 6 receptors to the Frizzled receptors, consequently keeping them in the inactive state of the WNT pathway. Proteins responsible for the phosphorylation of $\beta$-catenin in the inactive state of WNT were also more markedly expressed by increasing the degradation of cytoplasmic $\beta$-catenin, which may be elevated in the cytoplasm by non-activation of the pathway or by the increase in $\beta$-catenin mediated by seizures already described in the literature. Other mechanisms apparently related to this inactivation of the WNT pathway were evidenced, such as the negative regulation of the inhibitor of the intranuclear ligand of non-phosphorylated $\beta$-catenin, apparently seeking a compensation for the 
non-activation of the pathway. Possible manipulation of the WNT pathway can influence the molecular manifestations underlying seizures in brain tissue, being an effective target in preventing the worsening of the epileptogenic condition (29). Inhibition and factors such as GSK3b, for example, prevent $\beta$-catenin phosphorylation, thus allowing it to be translocated to the nuclear environment and transcribing genes from the activated WNT pathway, as well as DKK inhibition showing protective factors in relation to seizure control and neuronal death after an epileptogenic episode. Thus, the search for selective inhibitors based on the results of this analysis can serve as additional tools for the control of seizures in patients with type IIb FCD. The use of potential inhibitors of WNT pathway factors associated with anticonvulsant drugs can be investigated as possible therapeutic alternatives mainly by the association of these molecular and WNT alterations and their consequent interference in the normal tissue rearrangement after a seizure. Particularly in type Ilb FCD, the promotion of new alternatives to attenuate the refractoriness of patients before, and even after surgical resection, should be viewed with extreme enthusiasm and should be the subject of constant investigation.

\section{Declarations}

ETHICS APPROVAL AND CONSENT TO PARTICIPATE: This study was approved by the Comitê de Ética em Pesquisa (Research Ethics Committee) of the Pontifical Catholic University of Rio Grande do Sul (PUCRS).

CONSENT FOR PUBLICATION: All data published in this study was consented by the parents of the subjects.

AVALIABILITY OF DATA AND MATERIALS: The datasets generated and/or analysed during the current study are not publicly available in order to assure the privacy of the study subjects but are available from the corresponding author on reasonable request.

COMPETING INTERESTS: The authors declare no conflict of interest.

FUNDING: This study was financed in part by the Coordenação de Aperfeiçoamento de Pessoal de Nível Superior - Brazil (CAPES) - Finance Code 001.

AUTHORS CONTRIBUTION: DRM, GGZ, FACX, FM, and FVFR: molecular genetic studies and drafted the manuscript. DRM, EPN, ALFP, DCM, JGA and JCC conceived of the study, and participated in its design and coordination and helped to draft the manuscript. All authors read and approved the final manuscript.

ACKNOWLEDGEMENTS: The authors are grateful to CAPES-Brazil and the Pontifícia Universidade Católica do Rio Grande do Sul (PUCRS) for the scholarships for the authors.

ETHICAL PUBLICATION: We confirm that we have read the Journal's position on issues involved in ethical publication and affirm that this report is consistent with those guidelines. 


\section{References}

1. Bast T, Ramantani G, Seitz A, Rating D. Focal cortical dysplasia: prevalence, clinical presentation and epilepsy in children and adults. Acta neurologica Scandinavica. 2006;113(2):72-81.

2. Kabat J, Krol P. Focal cortical dysplasia - review. Pol J Radiol. 2012;77(2):35-43.

3. Palmini A, Najm I, Avanzini G, Babb T, Guerrini R, Foldvary-Schaefer N, et al. Terminology and classification of the cortical dysplasias. Neurology. 2004;62(6 Suppl 3):S2-8.

4. Blumcke I, Thom M, Aronica E, Armstrong DD, Vinters HV, Palmini A, et al. The clinicopathologic spectrum of focal cortical dysplasias: a consensus classification proposed by an ad hoc Task Force of the ILAE Diagnostic Methods Commission. Epilepsia. 2011;52(1):158-74.

5. Kuzniecky R. Epilepsy and malformations of cortical development: new developments. Current opinion in neurology. 2015;28(2):151-7.

6. MacDonald BT, Tamai K, He X. Wnt/beta-catenin signaling: components, mechanisms, and diseases. Dev Cell. 2009;17(1):9-26.

7. Pai SG, Carneiro BA, Mota JM, Costa R, Leite CA, Barroso-Sousa R, et al. Wnt/beta-catenin pathway: modulating anticancer immune response. J Hematol Oncol. 2017;10(1):101.

8. Pirone A, Alexander J, Lau LA, Hampton D, Zayachkivsky A, Yee A, et al. APC conditional knock-out mouse is a model of infantile spasms with elevated neuronal $\beta$-catenin levels, neonatal spasms, and chronic seizures. Neurobiology of disease. 2017;98:149-57.

9. Majolo F, Marinowic DR, Machado DC, Da Costa JC. Notch signaling in human iPS-derived neuronal progenitor lines from Focal Cortical Dysplasia patients. International journal of developmental neuroscience: the official journal of the International Society for Developmental Neuroscience. 2018;69:112-8.

10. Majolo F, Marinowic DR, Palmini ALF, DaCosta JC, Machado DC. Migration and Synaptic Aspects of Neurons Derived from Human Induced Pluripotent Stem Cells from Patients with Focal Cortical Dysplasia II. Neuroscience. 2019;408:81-90.

11. Parrini E, Marini C, Mei D, Galuppi A, Cellini E, Pucatti D, et al. Diagnostic Targeted Resequencing in 349 Patients with Drug-Resistant Pediatric Epilepsies Identifies Causative Mutations in 30 Different Genes. Human mutation. 2017;38(2):216-25.

12. Lee JM, Ramos EM, Lee JH, Gillis T, Mysore JS, Hayden MR, et al. CAG repeat expansion in Huntington disease determines age at onset in a fully dominant fashion. Neurology. 2012;78(10):690-5.

13. Brandenberger R, Wei H, Zhang S, Lei S, Murage J, Fisk GJ, et al. Transcriptome characterization elucidates signaling networks that control human ES cell growth and differentiation. Nature Biotechnology. 2004;22(6):707-16.

14. Mai M, Qian C, Yokomizo A, Smith DI, Liu W. Cloning of the human homolog of conductin (AXIN2), a gene mapping to chromosome 17q23-q24. Genomics. 1999;55(3):341-4. 
15. Hadjihannas MV, Brückner M, Behrens J. Conductin/axin2 and Wnt signalling regulates centrosome cohesion. EMBO reports. 2010;11(4):317-24.

16. Katoh M. WNT signaling in stem cell biology and regenerative medicine. Current drug targets. 2008;9(7):565-70.

17. Karasawa T, Yokokura H, Kitajewski J, Lombroso PJ. Frizzled-9 is activated by Wnt-2 and functions in Wnt/beta -catenin signaling. The Journal of biological chemistry. 2002;277(40):37479-86.

18. Liu Y, Zhang Z, Liang H, Zhao X, Liang L, Wang G, et al. Protein Phosphatase 2A (PP2A) Regulates EG5 to Control Mitotic Progression. Scientific reports. 2017;7(1):1630.

19. Liu C, Li Y, Semenov M, Han C, Baeg GH, Tan Y, et al. Control of beta-catenin phosphorylation/degradation by a dual-kinase mechanism. Cell. 2002;108(6):837-47.

20. Wang $C$, Wang $H$, Peng $Y$, Zeng B, Zhang Y, Tang $X$, et al. CTNNBIP1 modulates keratinocyte proliferation through promoting the transcription of $\beta$-catenin/TCF complex downstream genes. 2021;35(2):368-79.

21. Madsen TM, Newton SS, Eaton ME, Russell DS, Duman RS. Chronic electroconvulsive seizure upregulates beta-catenin expression in rat hippocampus: role in adult neurogenesis. Biological psychiatry. 2003;54(10):1006-14.

22. Rubio C, Rosiles-Abonce A, Trejo-Solis C, Rubio-Osornio M, Mendoza C, Custodio V, et al. Increase Signaling of Wnt/ $\beta$-Catenin Pathway and Presence of Apoptosis in Cerebellum of Kindled Rats. CNS Neurol Disord Drug Targets. 2017;16(7):772-80.

23. Theilhaber J, Rakhade SN, Sudhalter J, Kothari N, Klein P, Pollard J, et al. Gene expression profiling of a hypoxic seizure model of epilepsy suggests a role for mTOR and Wnt signaling in epileptogenesis. PloS one. 2013;8(9):e74428.

24. Cappuccio I, Calderone A, Busceti CL, Biagioni F, Pontarelli F, Bruno V, et al. Induction of Dickkopf-1, a negative modulator of the Wnt pathway, is required for the development of ischemic neuronal death. The Journal of neuroscience: the official journal of the Society for Neuroscience. 2005;25(10):264757.

25. Busceti CL, Biagioni F, Aronica E, Riozzi B, Storto M, Battaglia G, et al. Induction of the Wnt inhibitor, Dickkopf-1, is associated with neurodegeneration related to temporal lobe epilepsy. Epilepsia. 2007;48(4):694-705.

26. Hattiangady B, Shetty AK. Implications of decreased hippocampal neurogenesis in chronic temporal lobe epilepsy. Epilepsia. 2008;49 Suppl 5(0 5):26-41.

27. Morimoto K, Fahnestock M, Racine RJ. Kindling and status epilepticus models of epilepsy: rewiring the brain. Progress in neurobiology. 2004;73(1):1-60.

28. Yang J, Zhang $X$, Wu Y, Zhao B, Liu X, Pan Y, et al. Wnt/ $\beta$-catenin signaling mediates the seizurefacilitating effect of postischemic reactive astrocytes after pentylenetetrazole-kindling. Glia. 2016;64(6):1083-91.

29. Hodges SL, Lugo JN. Wnt/ $\beta$-catenin signaling as a potential target for novel epilepsy therapies. Epilepsy research. 2018;146:9-16. 


\section{Figures}

\section{Figure 1}

Image and electroencephalogram characteristics. (A) Magnetic Resonance Imaging (MRI) and EEG of Patient 1 showing the blurring and alteration of the cortical-subcortical interface of the posterior segment of the upper right frontal gyrus (arrow). (B) MRI and EEG of Patient 2 showing the change and lack of definition of the cortico-subcortical interface of the left occipital lobe (arrow) and electrographic onset crises in the occipital region on the right.

\section{Figure 2}

Graphic representation and results of gene expression. (A) Illustration of the methodology using brain tissue with and without dysplasia to compare the gene expression profile. (B) Heatmap of the comparison between patient 1 and patient 2 tissues represented by the color intensity difference (high green / low - red). The comparison between the $\triangle \mathrm{CT}$ values of each gene (greater or lesser differences in gene expression between tissues) can be presented using the scatter plot (C) or through the graphic representation in bars (D). (E) Graphs representing the analysis of gene expression through the application of the $2-\Delta \Delta C T$ formula of genes that presented fold change greater than 5 times.

\section{Figure 3}

Schematic representation of molecular alterations of the WNT pathway in the analyzed patients. Brain tissue from patients shows increased expression of KREMEN1 which increases DKK providing inactivation of Frizzled receptors by blocking the interaction with LPR5/6. The increased expression of AXIN2 and CSNK1A1 allows greater phosphorylation of $\beta$-catenin in the inactive phase of the WNT pathway, promoting an increase in its degradation. Besides, a compensatory mechanism may be associated with a reduction in the transcription of the CTNNBI1 gene, which is responsible for inhibiting the binding of TCF/LEF factors that promote the transcription of genes associated with the active WNT pathway and increase in the FZD9 gene transcript, possibly seeking secondary forms of WNT activation through new receptors. 